1913 , his red corpuscles numbered $3,900,000$ per cubic millimetre, the haemoglobin equalled 86 per cent., and the colour index was 1.1. This is one of the most successful cases I have had.

\section{Case Xr.}

A man, J. R., aged 58, was admitted to Chalmers Hospital on January 11th, 1913, suffering from moderately severe pernicious anaemia. His red corpuscles numbered $1,690,000$ per cubic millimetre, the haemoglobin equalled 48 per cent., and the colour index was 1.4. He has had two injections of neosalvarsan (January 14th, 1913, 0.5 gram; February 14th, 0.5 gram). Up to the present time the improvement in this case has only been slight, but sufficient time has not yet elapsed to allow of a definite statement regarding the result. On February 6th his red corpuscles numbered 2,490,000, the haemoglobin equalled 67 per cent., and the colour index was 1.2.

\section{Case XII.}

Another case, which is perhaps a case of pernicious anaemia, though the diagnosis is somewhat uncertain, is that of a man aged 42 He was admitted to Chalmers Hospital on December 16th, 1912, suffering from subacute combined paralysis and a moderate amount of anaemia (the red corpuscles numbered moderate amount of anaemia (the red corpuscles numbered cent., and the colour index was 1.4). The high colour index is very suggestive that the anaemia is of the pernicious type. He has had two injections of neo-salvarsan (December 23rd, 1912 0.4 gram ; January 24th, 1913, 0.4 gram). There has been a slight improvement in the condition of the blood, but no improvement in the nervous symptoms.

I mention this last case as I am anxious to give my full experience-every case in which I have employed this treatment-whether the result has been satisfactory or not.

I have now given the results of eleven cases of undoubted pernicious anaemia, and of one doubtful case treated by salvarsan; of the eleven undoubted cases, four have been apparently completely cured-whether in these cases a relapse will occur or not I am, of course, quite unable to say; in two cases there was very striking improvement; in one case there was very considerable improvement, but ultimately a relapse and death; in one case there was slight improvement and death from bronchopneumonia while under treatment; in two cases there was no improvement; and in one case slight im. provement, the patient being still under treatment. In the case of subacute combined paralysis associated with anaemia (perhaps pernicious anaemia), I tried the remedy as a last resource, though I did not expect it to beneficially affect the spinal lesion.

These results are very striking and very encouraging. I have had nearly forty years' experience of the ordinary arsenical plan of treatment of pernicious anaemia, and I have the greatest confidence in saying that the salvarsan treatment, so far as my present experience enables me to judge, is superior to the ordinary arsenical treatment. Further experience is, however, required before one can say whether the beneficial effects, which it undoubtedly produces in many cases, will be lasting or merely temporary.

In giving salvarsan I have always given it intramuscularly. In syphilis the usual way is to give it intravenously. In syphilis we know there is a definite organism, and we want to kill that organism as rapidly and as effectually as possible. In the treatment of syphilis the usual plan is to inject 0.6 gram of salvarsan into a vein. In primary and secondary syphilis the results are quite remarkable; relapses occur, however. The results in some of the tertiary cases are not so satisfactory as was at one time supposed. The results of salvarsan treatment in such conditions as tabes and generai paralysis of the insane-parasyphilitic lesionsare practically nil. There have been different opinions expressed on this point, but I think the experience of the great majority of reliable physicians is that in tabes and general paralysis of the insane salvarsan is of little or no use.

In pernicious anaemia I have employed the intramuscular method of administration, for, in this disease, we want, I think, a remedy which will produce sustained and continued effects. We do not know what the toxin is which appears to be the cause of pernicious anaemiawhether a germ toxin or a chemical toxin. The dose I have a!ways employed has been 0.3 gram, half the dose which is usually given in syphilis. In severe and advanced cases of pernicious anaemia, in which the patient's hold of life is very precarious indeed - and this was the condition in many of the cases I have brought before you-a very little thing will turn the scale. I have therefore hesitated in these cases to give the remedy by the intravenous method.

In some cases the salvarsan, when given intramuscularly, produces very considerable inflammation, pain, and swell. ing; but the neo-salvarsan seems to be much more satis. factory in this respect-it does not produce such marked local results. In the cases in which I have used neo. salvarsan I have found that there is little or no local pain or hardness, and little or no rise in temperature. In the meantime I shall continue to give the remedy intra. muscularly.

\section{ADDENDUM (May $z t h, 1913)$.}

CASE IX.

This patient was readmitted to Chalmers Hospital on March 3 rd, 1913, suffering from a severe relapse. The red blood corpuscles numbered $1,010,000$ per cubic millimetre; haemoglobin 36 per cent.; colour index 1.7 . As the result of two globin 36 per cent.; colour index 1.7. As the result of two
injections of salvarsan he has improved very rapidly; I fear, however, that as soon as he is again discharged another relapse will in all probability occur, and that the effects of the treatment will be merely temporary.

\section{CASE XIr.}

During February, 1913, this patient rapidly got worse, the paralysis passing in to the flaccid stage. He died on March 22 nd. Post-mortem examination showed all the typical appearances of pernicious anaemia.

\section{ACUTE PERFORATION OF THE GALL BLADDER :}

WITH AN ACCOUNT OF SIX CASES.

BY

L. R. BRAithwaite, M.B., Ch.B.Vict., F.R.C.S.Eng. HONORARY ASSISTANT SURGEON, LEEDS GENERAL INFIRMARY, ETC.

In these days, when the early and exact diagnosis of acute abdominal conditions is a matter of extreme importance, it is essential to form a clear conception of the characteristic signs and of the methods of treatment of even the rarest forms of abdominal catastrophe.

Although McWilliams ${ }^{1}$ and Cotte and Arnaud ${ }^{2}$ have given such excellent descriptions of perforation of the gall bladder, in nearly all its varieties, there are perhaps some points of particular interest in the cases given below.

The effect of sterile bile in the general peritoneal cavity is to destroy the bactericidal quality of serum.

In rabbits Noetzel showed that whereas a certain amount of Staphylococcus aureus or Bacillus coli or streptococci placed in the normal healthy peritoneal cavity was innocuous, a similar amount was always fatal pro. vided the bile from the healthy gall bladder was allowed to escape into the body cavity.

Normal bile is well tolerated for a considerable time, and becomes gradually shut off by inflammatory adhesions.

In a case recorded by Garré, operation undertaken two months after the injury which ruptured the gall bladder showed a localized and large collection of bile; the patient got well. In a case recorded by Hildebrandt operation was successful twenty-three days after a tear into the hepatic duct.

Bile which escapes from a rupture in a gall bladder containing stones is not an aseptic fluid, it teems with the Bacillus coli or other organisms.

It is in cases in which gangrene and rupture occur in an infected gall bladder that rapid and fatal peritonitis develops. Clearly the prognosis depends chiefly upon the number, character, and virulence of the organisms present in the bile.

McWilliams, omitting the purely traumatic variety, describes three kinds of rupture of the gall bladder :

1. Rupture from over-stretching; with or without stones.

2. Perforation from pressure of stone on the wall.

3. Perforation from gangrene due to :

(a) Thrombosis of vessels with or without stones.

(b) Cutting off of circulation from pressure of stone.

(c) Diphtheritic, ulcerative infection of the wall with or without stone. 
To these there must probably be added :

4. Subperitoneal perforation of the gall bladder with subperitoneal extravasation of bile as illustrated in Case vi.

Signs and Symptoms.

As Sir Berkeley Moynihan has frequently pointed out, in every case of gall-bladder trouble, provided the history be carefully noted, there is a complaint of flatulent indigestion over a long period.

Attacks of severe pain in the region of the gall bladder, with shivering fits of great or small intensity, may be present in nearly all cases where there is an infection of any degree of severity.

It is of great interest to find that the sudden agonizing pain characteristic of the rupture of a hollow viscus, followed by a zealous rigidity of the neighbouring body wall, does not occur in all cases; probably both conditions depend to some extent upon the virulence of the invading bile.

At the moment of perforation there is always severe pain in the region of the gall bladder, followed in two or three hours by pain referred to the umbilicus. Moynihan showed how the fluid from a perforated duodenal ulcer gravitates over the bend of the hepatic flexure to the right iliac fossa; fluid escaping from the gall bladder follows the same course, and gives rise to swelling and pain in the region of the appendix.

It is a matter of much interest and of some importance to note that the effusion of a quantity of bile of a degree of virulence sufficient to cause a peritonitis will in course of a day or two give rise to a definite fluid swelling simu. lating in every way an appendix abscess (Case v).

The degree of rigidity is most variable and there is hardly ever the "marble" hardness found in other abdominal catastrophes. In none of the cases seen by the writer has there been that degree of involuntary stiffness and inability to move about seen in other grave abdominal conditions.

It would appear that the absorption of bile from the peritoneal cavity slows the pulse-rate, just as absorption of bile in cases of jaundice reduces the normal pulse-rate. The pulse-rate after twenty-four hours will depend upon the virulence of the infection and the kind of peritonitis. The temperature may remain subnormal until the formation of an abscess or the onset of acute peritonitis.

\section{Treatment.}

Early operation naturally gives the best results. If one can be certain that a catastrophe has occurred, as evidenced by a clear history and by the presence of extensive rigidity in the right hypochondrium, operation should be under taken at once, before a rise in pulse-rate indicates a considerable extent of peritonitis. The presence of dullness in the right flank would necessitate immediate operation. To be able to say with certainty that there is a perforation of the gall bladder in the early stage, and not a condition of cholecystitis, is probably impossible. So long as the rigidity does not extend beyond the neighbourhood of the gall bladder and so long as the pulse-rate keeps below 95 the case should be watched.

It is of great assistance in helping to come to this decision if the patient lies easily and placidly in his bed and moves about in comfort.

It may happen (Case v) that the condition rapidly settles down within a few hours, and in a few days there is left a localized, dull, tender swelling in the appendix region.

The incision must be vertical, splitting the rectus of the right side. It is an easy matter to extend the incision up or down as required.

In Case IV there was well-marked oedema of the subcutaneous tissues and staining by bile. To quote McWilliams :

He who is the keenest observer of the conditions found in the abdomen, who can best estimate the virulence of the infection present, the degree of adhesions, and the pathological conditions present in the gall-bladder system, who can make a good mental estimate of how much or how little the patient can stand, who can get in the quickest, inflicting the least possible trauma, will have the greatest successes in these trying cases.

If the gall bladder can be easily and quickly removed, this should be done in every case where there is gangrene present. A large maroon gall bladder with a perforation

and containing stones may be emptied and drained after widening the perforation. If there be a stone in the cystic duct, the gall bladder should be removed if circumstances permit (Case rv).

A small contracted gall bladder is difficult to remove at any time, and it would be well in most cases to open, empty, and drain rather than make any attempt to remove. In many cases it is quicker to remove the gall bladder than to drain it. Mere suture of the perforation should never be attempted.

Cholecystotomy gives the best immediate results, 60 per cent. recovering; probably in expert hands cholecystectomy will give the best permanent results.

In all cases the right kidney pouch and the area of the cystic duct must be drained, either by stab puncture through the right loin or from the anterior wound.

If the whole abdomen is flooded with bile, as much of it as can be mopped up by hot swabs should be removed and a suprapubic tube (inserted through a separate puncture) should be pushed to the bottom of the pelvis. In every case Murphy's rectal infusion in the Fowler position must be the after-treatment.

Where the bile has collected in the right iliac fossa, in the form of an appendix abscess (Case v), the incision should be lengthened downwards, the bile and pus mopped out, and the area drained by tube through a puncture over the right anterior superior spine or through the anterior wound.

Whatever the procedure adopted it must be gently and quickly carried out. Washing out the peritoneal cavity cannot be done effectually at any time or under any con. ditions, and should never be attempted. The condition of the patient may be so grave (Case IV) that very rapid drainage must be obtained and removal of stones from the gall bladder left for a subsequent operation.

\section{Statistics.}

In 3,180 cases operated upon for gall stones (McWilliams) there were 29 cases of acute perforation. In 1,000 cases operated upon for gall stones at the Leeds Infirmary there were 10 acute perforations, 1 being a perforation of the hepatic duct. McWilliams records 108 cases of perforation, and to these the cases in the writer's list must be added, and 2 cases published in Sir Berkeley Moynihan's book, Gallstones.

CASE I.

Seen by Sir Berkeley Moynihan in consultation with Dr. Hind of Harrogate. The writer assisted at the operation.

Mr. J., aged 60, had resided in Ceylon for thirty years. Enjoyed good health until three years ago, when he became dyspeptic, constipated and depressed. Two and a half years ago he developed diverticulitis of the sigmoid with obstruction and abscess, and underwent an operation.

During the time of this illness he complained of intense pain in the right hypochondrium, and it was feared there might be some further suppuration of the nature of that dealt with at the operation for diverticulitis. He was treated with $B$. coli vaccines, operation for diverticulitis.

In April, 1912, he had a return of his old trouble, dyspepsia and constipation, and was treated by a pill of aloes and nux vomica.

Suddenly on April 30th he was seized with great colicky pain and sickness, and for twelve hours could not locate the trouble. The abdomen was much distended, but there was no marked resistance. He then had rigors, the temperature reaching $106^{\circ} \mathrm{F}$. Distinct tenderness over the gall bladder developed, and on May 1st Sir Berkeley Moynihan saw him and decided to wait.

On May 4th Sir Berkeley operated. An incision was made vertically over the upper part of the right rectus, the muscle being split longitudinally. Much free golden yellow bile escaped. The area of the gall bladder was shut off by huge masses of intensely engorged omentum which were adherent by lymph to each other and to the liver and gall bladder.

After separating the adhesions a small circular perforation was seen, about $2 \mathrm{~mm}$. in diameter, on the inferior surface of was seen, about $2 \mathrm{~mm}$. in diameter, on the inferior surface of
the fundus of the gall bladder. The walls of the gall bladder were thinned away at this part so that the edges of the perforation were thin as tissue paper, and were stained a brilliant orange red. Yellow bile leaked in a slow stream from the opening. The aperture was enlarged, many small stones were removed, and a tube fixed into the oall bladder. There was no a have been difficult. The area of the right kidney was drained and the abdomen closed.

The patient made an uninterrupted recovery.

Collinson.

Mr. E. O, aged 54. At 4 a.m. on October 24th he was seized with a sudden umbilical pain. He could not rest, got up, 
partially dressed, and went dowhstairs: He had bad pain all day long, and vomited once. Next night he had very little sleep, and several attacks of colicky pain in the body ; opium was given. Next morning (October 25th) the pain was no better, abdomen getting swollen, bowels not open. At 3.30 p.m. in the upper zone. Liver dullpess present.

The history, as far as he was able to give it, was that he had been for some years subject to attacks of pain in the upper abdomen, not related to food, and associated with vomiting. He was a painter by trade, but there was no blue line and the knee-jerks were present.

The diagnosis of perforation into the peritoneum (? nature) was made.

Operation.

Incision vertically over the right rectus, about opposite the umbilicus. Appendix area explored and found normal; marked spasm of the small intestines; some free bile seen at the root of the mesentery. Incision lengthened upwards, and much bile found free in the peritoneal cavity; only one flake of mucopus noted. The gall bladder was small and very adherent to the punentum; there was a small perforation of the under part at omentum; there was a small perforation of the under part at
the fundus, at a spot where there were no omental adhesions. The opening was enlarged and many stones removed, and the gall bladder was drained. Large tube drain through a separate opening in the right kidney pouch

The patient recovered and went home on November 16th.

CASE III.

Operation at Leeds General Infirmary by Mr. Collinson.

G. H. C., a man aged 62, was in a most wretched condition on admission, and was incapable of giving any history whatever.

In addition to the abdominal condition he had marked aortic stenosis, and operation was undertaken under intraspinal injection of stovaine.

Temperature on admission was $99^{\circ}$, pulse 110 , and respirations 33 . Abdomen very distended, and moved very slightly on respiration.

Operation was performed at once (May 1st). A long incision was made over the right rectus; there escaped a quantity of yellow fluid, which was part of a collection around the gall bladder. This collection was shut off by adhesions of small intestines. A few small gall stones were found loose in the peritoneal cavity. There was a small perforation on the under surface of the gall bladder. The gall bladder was drained by tube, and the area of peritoneum invaded by bile was drained by another tube. The patient lived until May 9 th

Post-mortem, marked aortic stenosis, coronary atheroma, fat necrosis, and general peritonitis were found.

The three following cases were operated upon by the writer.

CASE IV.

Seen in consultation with Dr. Hall of Dewsbury.

Mr. M., aged 64, had some history of indigestion, but none of severe abdominal pain at any time. On April 25th, 1912, he was feeling a bit "run down," with vague abdominal discomfort. During that night he woke with severe pain in the body, and on the morning of April 26th Dr. Hall saw him. At that time he had very severe pain all over the body and was rolling about the bed in agony, the abdomen was markedly distended and very tender all over, the pulse was 120 per minute, and the bowels were open. On April 27th there was definite localized tenderness over the appendix and less pain and tenderness elsewhere.

I saw him on April 28th. He was a very stout, plethoric man, and he lay in bed on his back with his knees drawn up and apparently in comfort. His face and lips were livid and he had just a suggestion of jaundice. His abdomen was enormously distended and respiratory movements were only slight. There was general tenderness, but definite, localized, exquisite tenderness over McBurney's point. There was dullness in the right flank and a fluid thrill over the right half of the abdomen. The pulse was small and 130 per minute. Rectal examination revealed some fullness high up on the right side. Liver dullness was normal and he had not had right side. Liver dullness was normal and he had not had
a rigor. "Appendicitis with peritonitis" was diagnosed and possibly a large abscess.

\section{Operation.}

Immediate operation was decided upon. He was far too ill to be removed, and only very limited accommodation was availincision was made over the lower part of the right rectus and the muscle split. In cutting through the fat and deep fascia it was noticed, and remarked upon, that there was oedema of the tissues and obvious staining by bile pigments.

On opening the peritoneum a very large quantity of yellow bile mixed with mucus and purulent exudate escaped, the mixture looking like sellow pea-soup. In it were many small, faceted black gall stones. The incision was rapidly lengthened up to the costal margin, and large quantities of thick yellow fluid escaped, with dozens of gall stones. Innumerable small stones but much flakry lymph and stringy mucus.

The gall bladder was one and $a$ half times the natural size, and was intensely red. On its lower surface, near the fundus, was a small circular perforation about $3 \mathrm{~mm}$. in diameter; its edges were a brilliant golden yellow. Large numbers of stones lay in the gall bladder and others in the cystic duct. The perforation was enlarged by cutting with scissors up to the fundus and as many loose stones as could be got quickly out were removed. A tabe about 9 in. in diameter was stitched into the gall bladder. Some stones were picked off the intestines, but many were left, and much fluid was mopped away. From the lowest part of the wound a long, wide tube was placed as a drain to the bottom of the pelvis, and the wound closed in layers.

He was given continuous rectal infusion and fixed in the Fowler position. He made an uninterrupted recovery. The whole operation occupied thirty-five minutes. Large numbers of stones came through the tube, and for many months he healed.

It is nearly certain that had I attempted to remove the gall bladder in this case or to remove the stones in the cystic duct he would have died on the table. It was of great interest to note that some weeks after the operation the patient explained that the very first onset of his pain had been over the region of the gall bladder.

CASE v.

Seen in consultation with Dr. Orford, Pontefract.

Mr. W., aged 60 , for many years had had bad indigestion, characterized by distension and frequently relieved by viting. On several occasions he had severe pains under the right ribs and occasionally some shivering. He had been jaundiced on one occasion.

Dr. Orford saw him on October 16th, 1912, and found him suffering from bad pain over the gall bladder and occasional vomiting. There was marked localized tenderness and some rigidity. Pulse was 80 per minute, temperature was $100^{\circ} \mathrm{F}$., and respirations 30

On October 17th he was worse, the pulse had gone up to 85 , and the temperature had fallen to normal, but he looked ill. He complained of a catchy pain in the right ribs when he breathed deeply, but he easily rolled about in bed and sat up to have the back of his chest examined. There was definite
rigidity over the upper half of the right side of the abdomen and exquisite tenderness. The right flank was resonant. The diagnosis of acute cholecystitis was made.

On October 18th he was removed to a nursing home in Leeds in much the same condition. Perforation of the gall bladder was now definitely suspected.

On October 19th he was better, the pulse falling to 80 and the temperature being normal. The rigidity and tenderness were restricted to the gall bladder region.

On October 20th there was some bile in the urine and he was slightly jaundiced. The bowels were opened and the tenderness over the gall bladder was less marked. There was tenderness and some rigidity over the appendix area.

From October 21st to 23 rd he improved daily and a definite lump gradually made itself apparent in the appendix region, the size of an orange. Temperature rose gradually to $100^{\circ} \mathrm{F}$. Tenderness over the gall bladder less marked.

Operation.

On October 24th an incision was made vertically over the middle half of the right rectus, splitting the muscle. There were recent plastic adhesions and intense engorgement of the intestines. On separating the adhesions clear bile escaped from the region of the gall bladder. The incision was lengthened up to the costal margin.

The gall bladder was of normal size, but thick and deep red in colour. On the under surface near the fundus was a cleancut circular perforation $3 \mathrm{~mm}$ in diameter, its edges pigmented a golden yellow. From it exuded clear yellow bile and some stringy mucus. Cholecystectomy was easily performed; the gall bladder contained many small faceted stones. Three or four spots of fat necrosis were noticed in the omentum. A large drain, reaching the stump of the cystic duct and the right kidney pouch, was introduced. Examination nearer the kidney pouch, was introduced. Examination nearer the appendix region revealed a large collection of bile and pus, not
unlike pea-soup, shut off on the lower, inner, gnd outer sides by recent adhesions, but open to the upper side by a channel leading up on the outer side and in front of the ascending colon to the gall bladder. Along the channel was yellow bile. The lowest point of the wound.

He made an uninterrupted recovery.

CaSE vi.

When admitted to the Leeds General Infirmary the patient was most gravely ill, pulse 160 per minute, respirations 36 , tem. perature $101.5^{\circ} \mathrm{F}$. He was a very big, stout man, aged 49 , and was unable to give much history. He said he had pain all over the body and was vomiting frequently. For many years he had the bod from pains under the front of the ribs on the right suffered from pains undertion. Fourteen days before admission side and constant indigestion. Fourteen days before admission he had a rigor, but no very bad pain; seven days later he began
to have bad pain over the gall bladder which spread all over his body.

The abdomen enormously distended, moving well except over the right hypochondrium. There was exquisite tenderness over the gall bladder where there was some resistance suggesting a solid mass. Diagnosis of acute cholecystitis with possibly perforation was made. 
Operation.

An incision was made vertically over the upper part of the right rectus splitting the muscle. A little clear free fluid escaped from the peritoneal cavity, but no bile. Plastic adhesions between coils of congested and distended intestine, confined to the right hypochondrium. Stomach, duodenum, hepatic flexure of colon adherent to the gall bladder by plastic Jymph

On separating the adhesions, the gall bladder was seen to be twice its usual size, deep maroon in colour, and presented five or six patches of black gangrene over which the peritoneal coat was intact and shiny. The patches of gangrene were each about the area of a sixpence.

The most remarkable appearance was over the region of the common duct, where there was a large swelling the size and shape of a banana looking like a full leech or a coil of gangrenous intestine; behind it the finger could be easily passed into the foramen of Winslow. There was no perforation into the general peritoneal cavity and no free bile.

The gall bladder was quickly and easily removed, and an incision made into the peritoneum over the position of the common duct. Free black bile escaped, probably about an ounce. The common duct lay in its usual position and was pale position and was pale perforation of it could perforation of it could its usual size and lay free, stripped of its peritoneal coat by the layer of bile. digaram.

The region of the cystic duct was crained, and the right kidney pouch was drained separately by a large ight loin. The ound was closed.

The whole operation occupied thirtyive minutes. He

was most gravely ill throughout, and he died some little time after the abdomen was closed.

\section{Post-mortem Examination.}

There was very rapid decomposition ; the common duct was reduced to a black-green pulpy mass, but there was no break in its continuity and no sign of a perforation. No stones were found.

There was a small recent abscess in the upper pole of the right kidney. The pancreas was soft and almost diffuent

The gall bladder contained twenty-two stones. The wall was thick and intensely red. There were ten definite areas where the mucous membrane was black and thinned away until in some parts the peritoneum only remained.

In all the part covered by peritoneum there was no perforation, and none could be found in the portion devoid of peri toneal covering; curiously enough there was not a single patch of gangrene on this portion of the organ.

From one of the patches of gangrene on the under surface near the fundus, starting from the mucous surface, a probe could be passed under the peritoneal coat towards the cystic duct and along it.

It is a most unfortunate fact that the specimen was misplaced by the Pathological Department and cannot be found.

It is difficult to account for the presence of bile under the peritoneal covering of the common duct except on the grounds of a perforation. Whilst it was unfortunate the common duct underwent such rapid decomposition, it was not so bad but that one could come to a definite decision that no leakage had occurred from it. Gangrene of the duct was not present at the time of operation, because the duct could be seen looking pale red when the black bile had drained away.

Is it possible that bile had passed down from the gall bladder, through the breach in the mucous membrane at the gangrenous spot, along the track where the probe passed, and so to the cystic and common ducts?

It would appear no explanation other than a subperitoneal perforation of the gall bladder can be offered.

My thanks are due to Sir Berkeley Moynihan and to Mr. Collinson for giving me permission to use their cases.

1 Annals of Surgery, FebruFERENCES

ebruary, 1912. 2 Clinique Chirurgicale de

\section{THE INCIDENCE OF OPHTHALMIA NEONA-} TORUM IN LONDON.

By N. BISHOP HARMAN, M.A., M.B.Cantab., F.R.C.S:ENG.,

LECTURER IN OPHTHALMOLOGY TO THE WEST LONDON POST-GRADUATE COLLEGE; ASBISTANT OPHTHALMIC SURGEON TO THE WEST LONDON HOSPITAL; OPHTHALMIC SURGEON TO THE
BELGRAVE HOSPITAL FOR CHILDREN.

The first official statement on the incidence of purulent conjunctivitis in the newborn in London appears in the recently issued report of the Medical Officer of Health of the London County Council. The report is for the year 1911, and it bears the printed date of February 18th, 1913.

The information published in the report is the outcome of the order which made this disease notifiable within the London County area. This was done in March, 1911, by an order extending the provisions of Section 55 of the Public Health (London) Act, 1891, to ophthalmia neonatorum. Prior to this date there was no authoritative knowledge of the incidence of the disease in the population at large, and it was spoken of as "rare" or " common," just as cases bulked largely or otherwise in the minds of medical men and social workers who came in contact with them in maternity hospitals and in private practice. It was obviously impossible to obtain general information for a whole community without official action. Indeed, no attempt seems to have been made to obtain such informa. tion except "on one occasion, when by the courtesy of several doctors in private practice in one quarter of London I was able to gather and publish statistics concerning some 12,000 births. $^{1}$ It will be of interest to put these private returns made in 1906 alongside the official returns for 1911.

The Council's notification order came into force on March 13th, and from that date until the end of the year 673 cases were notified (p. 82). The births in London for 1911less stillbirths (corrected)-are given (p. 6) as 100,830. To obtain the percentage incidence of the disease it will be necessary to compute the probable figures of the disease for the whole year, instead of as is given, for nine and a half months. There is no reason to suspect any material variation in the incidence of the disease in different seasons of the year. The corrected figures for the year would be 850 cases. Compared with the number of births given, that means an incidence of 0.843 per cent. This figure is very nearly the same as that obtained in my privato census for 1906; in 12,680 live births there were 110 cases of purulent conjunctivitis, or an incidence of 0.867 per cent.

The point of greatest importance is the number of cases in which the disease is productive of injury to or loss of sight. The Council's report states that the condition of 278 cases occurring in the practice of midwives was followed out so that in most the ultimate issue was known. There was a wastage, however; 17 children died whilst suffering from the disease, and in 30 instances the parents removed, and could not be traced. There are left 231 cases; of these, 218 were reported to be completely cured; in 13 cases there was impairment of vision.

The rate of injury is, therefore, 13 in 231 , or 5.6 per cent. It is interesting to compare this with the private return. In that I found 6 children with permanently damaged eyes out of 110 affected, or an injury-rate of 5.4 per cent.

Details of the 13 cases in which the sight was affected are given :

$\begin{array}{lcccc}\text { Blind in both eyes } \ldots & \ldots & & & \\ \text { Blind in one eye, other damaged } & \ldots & & 3 \\ \text { Blind in one eye, other uninjured } & \ldots & \ldots & 1 \\ \text { Both eyes damaged } \ldots & \ldots & \ldots & \ldots & 2 \\ \text { One eye damaged } \ldots & \ldots & \ldots & \ldots & 3\end{array}$

There was a history of vaginal discharge in the mother in 7 of these cases. In every case except one the eyes were cleansed with some antiseptic immediately or soon after birth; where there was delay it was due to the birth of the child before the arrival of the midwife. In the single case in which cleansing is not noted the disease did not appear until the eleventh day after birth, so that it is almost certainly a secondary infection. Efficient medical help was obtained with promptitude in most cases. There was delay of three, five, and, in one case, ten days, owing 